\title{
Lactobacillus plantarum Exhibits Antioxidant and Cytoprotective Activities in Porcine Intestinal Epithelial Cells Exposed to Hydrogen Peroxide
}

\author{
Jing Wang $\mathbb{D}^{1,2}$ Wei Zhang, ${ }^{1,2}$ Sixin Wang, ${ }^{1,2}$ Yamin Wang, ${ }^{1} \mathrm{Xu} \mathrm{Chu,}{ }^{1,3}$ and Haifeng Ji ${ }^{1,2}$ \\ ${ }^{1}$ Institute of Animal Husbandry and Veterinary Medicine, Beijing Academy of Agriculture and Forestry Sciences, \\ 100097 Beijing, China \\ ${ }^{2}$ Sino-US Joint Laboratory of Animal Science, Beijing Academy of Agriculture and Forestry Sciences, 100097 Beijing, China \\ ${ }^{3}$ College of Agriculture, Qinghai University, 810016 Xining, China
}

Correspondence should be addressed to Jing Wang; wangjing976119@126.com

Received 22 May 2021; Accepted 12 July 2021; Published 31 July 2021

Academic Editor: Tongxing Song

Copyright (c) 2021 Jing Wang et al. This is an open access article distributed under the Creative Commons Attribution License, which permits unrestricted use, distribution, and reproduction in any medium, provided the original work is properly cited.

Probiotics are widely used for protection against stress-induced intestinal dysfunction. Oxidative stress plays a critical role in gastrointestinal disorders. It is established that probiotics alleviate oxidative stress; however, the mechanism of action has not been elucidated. We developed an in vitro intestinal porcine epithelial cells (IPEC-J2) model of oxidative stress to explore the antioxidant effect and potential mode of action of Lactobacillus plantarum ZLP001. The IPEC-J2 cells were preincubated with and without $L$. plantarum ZLP001 for $3 \mathrm{~h}$ and then exposed to hydrogen peroxide $\left(\mathrm{H}_{2} \mathrm{O}_{2}\right)$ for $4 \mathrm{~h}$. Pretreatment with $L$. plantarum ZLP001 protected IPEC-J2 cells against $\mathrm{H}_{2} \mathrm{O}_{2}$-induced oxidative damage as indicated by cell viability assays and significantly alleviated apoptosis elicited by $\mathrm{H}_{2} \mathrm{O}_{2}$. L. plantarum ZLP001 pretreatment decreased reactive oxygen species production and the cellular malondialdehyde concentration and increased the mitochondrial membrane potential compared with $\mathrm{H}_{2} \mathrm{O}_{2}$ treatment alone, suggesting that L. plantarum ZLP001 promotes the maintenance of redox homeostasis in the cells. Furthermore, L. plantarum ZLP001 regulated the expression and generation of some antioxidant enzymes, thereby activating the antioxidant defense system. Treatment with L. plantarum ZLP001 led to nuclear erythroid 2-related factor 2 (Nrf2) enrichment in the nucleus compared with $\mathrm{H}_{2} \mathrm{O}_{2}$ treatment alone. Knockdown of Nrf2 significantly weakened the alleviating effect of L. plantarum ZLP001 on antioxidant stress in IPEC-J2 cells, suggesting that Nrf2 is involved in the antioxidative effect of L. plantarum ZLP001. Collectively, these results indicate that L. plantarum ZLP001 is a promising probiotic bacterium that can potentially alleviate oxidative stress.

\section{Introduction}

Oxidative stress is caused by an imbalance between prooxidants and antioxidants and is implicated in extensive human and animal diseases. Oxidative stress is often associated with the accumulation of reactive oxygen species (ROS), which can induce DNA hydroxylation, protein denaturation, and lipid peroxidation [1] and thus compromise the viability of cells, ultimately causing several diseases [2]. The intestine is more vulnerable to oxidative stress owing to its continuous exposure to the luminal environment. Intestinal oxidative stress influences the digestibility and absorption of nutrients and can cause various intestinal diseases [3, 4]. In particular, during the critical life phases of animals, such as weaning, the underdeveloped intestine combined with depressed intake can lead to the insufficient synthesis of endogenous antioxidants. Therefore, antioxidant supplementation strategies have been considered. Dietary antioxidants, such as vitamins $\mathrm{C}$ and $\mathrm{E}$, and metals, such as $\mathrm{Zn}$ and $\mathrm{Cu}$, can neutralize oxidative molecules and play an important role in maintaining redox homeostasis in humans and animals $[5,6]$.

Probiotic bacteria have been shown to exhibit antioxidant activity both in vitro and in vivo [7-9]. Several probiotic strains and their products present in food exert remarkable 
antioxidant activities, and these strains exhibit high viability in anaerobic environments, have a strong oxygen radicalscavenging ability, and produce several antioxidant enzymes [10-12]. Antioxidative properties vary widely among bacterial strains, indicating that they are strain specific $[7,13]$. Probiotics have been demonstrated to exert antioxidant activities in various host cells and the human body by modulating the redox status by scavenging free radicals, chelating metal ions, regulating enzymes, and modulating the intestinal microbiota $[8,9]$. Further, it has been reported that probiotics exert antioxidant activity mainly through the induction of detoxifying enzymes via the activation of transcription factor nuclear erythroid 2-related factor 2 (Nrf2) $[14,15]$. Other regulatory pathways involving Sirt1, MAPK, and PKC, which may trigger the dissociation of Nrf2 or enhance the cell homeostasis, are also involved in the regulation of their antioxidant action [9]. However, several questions regarding the underlying mechanisms of the antioxidative roles of probiotics, such as concentration effects, mitochondrial function, and Nrf2 dissociation pattern, remain unsolved.

In our previous studies, we demonstrated that L. plantarum ZLP001 isolated from healthy piglet ileal mucosa [16] exerts a strong antioxidant ability, is highly viable in hydrogen peroxide, has a high oxygen radical-scavenging ability in vitro, and alleviates oxidative stress in weaning piglets in vivo [17]. However, the antioxidant capacity of L. plantarum ZLP001 under oxidative stress or its mechanisms of action is not well understood. Therefore, in this study, we evaluated the effect of $L$. plantarum ZLP001 pretreatment in an in vitro model of oxidative stress using porcine intestinal epithelial cells (IPEC-J2) treated with hydrogen peroxide $\left(\mathrm{H}_{2} \mathrm{O}_{2}\right)$.

\section{Materials and Methods}

2.1. Bacterial Strain, Cells, and Culture Conditions. L. plantarum ZLP001 was originally isolated in our laboratory, from the ileal mucosa of healthy piglets $4 \mathrm{w}$ after weaning. The strain was identified by the China Center of Industrial Culture Collection (Beijing, China) and is preserved in the China General Microbiological Culture Collection Center (CGMCC No. 7370). L. plantarum ZLP001 cells were cultured in improved de Man, Rogosa, and Sharpe liquid medium (10 g peptone, $5 \mathrm{~g}$ yeast powder, $20 \mathrm{~g}$ glucose, $10 \mathrm{~g}$ beef extract, $5 \mathrm{~g}$ sodium acetate, $2 \mathrm{~g}$ ammonium citrate dibasic, $2 \mathrm{~g}$ dipotassium phosphate, $0.58 \mathrm{~g}$ magnesium sulfate, $0.19 \mathrm{~g}$ manganese sulfate, $1 \mathrm{~mL}$ of Tween-80, and water to $1,000 \mathrm{~mL} ; \mathrm{pH} 6.5$ ) at $37^{\circ} \mathrm{C}$ under anaerobic conditions.

The porcine intestinal epithelial cell line (IPEC-J2) was a generous gift from Dr. Glenn Zhang (Oklahoma State University, Stillwater, OK). The IPEC-J2 cells were cultured in DMEM/F12, a 1:1 mixture of Dulbecco's modified Eagle's medium and Ham's F-12 (Gibco ${ }^{\mathrm{TM}}$, Thermo Fisher Scientific, Waltham, MA, USA) supplemented with $10 \%$ fetal bovine serum (FBS; $\left.\mathrm{Gibco}^{\mathrm{TM}}\right)$, streptomycin $(100 \mu \mathrm{g} / \mathrm{mL})$, penicillin $(100 \mathrm{U} / \mathrm{mL})$, and $1 \%$ ITS premix $(5 \mu \mathrm{g} / \mathrm{mL}$ insulin, $5 \mu \mathrm{g} / \mathrm{mL}$ transferrin, $5 \mathrm{ng} / \mathrm{mL}$ selenium; ScienCell, San Diego, CA) at $37^{\circ} \mathrm{C}$ in a $5 \% \mathrm{CO}_{2}$ and $95 \%$ air atmosphere with $90 \%$ humidity.

2.2. Oxidative Stress Model Establishment. An in vitro oxidative stress model was established by treating IPEC-J2 cells with $\mathrm{H}_{2} \mathrm{O}_{2}$. Cell viability was assessed using the methyl thiazolyl tetrazolium (MTT) assay. IPEC-J2 cells were seeded in 6-well tissue culture plates (Costar, Corning Inc., Corning, NY, USA) at $2.5 \times 10^{5}$ cells/well and cultured overnight with $2 \mathrm{~mL}$ of complete culture medium. After treatment with $\mathrm{H}_{2} \mathrm{O}_{2}$ at final concentrations of $0,250,500,750,1000,1250$, 1500 , and $1750 \mu \mathrm{mol} / \mathrm{L}$ for $4 \mathrm{~h}$, the cells were incubated with $5 \mathrm{mg} / \mathrm{mL}$ of MTT working solution at $37^{\circ} \mathrm{C}$ for $4 \mathrm{~h}$. The absorbance at $490 \mathrm{~nm}$ was measured using a Multiskan FC instrument (Thermo Fisher Scientific, Waltham, MA, USA). The median lethal dose (LD50) of $\mathrm{H}_{2} \mathrm{O}_{2}$ was calculated by probability unit and the optimal concentration of $\mathrm{H}_{2} \mathrm{O}_{2}$ was selected to establish the IPEC-J2 cell-based oxidative stress model.

2.3. Cell Treatments. IPEC-J2 cells were seeded in 6-well tissue-culture plates at $2.5 \times 10^{5}$ cells/well. After overnight culture, the complete culture medium was replaced with culture medium without antibiotics, and the cells were incubated with $L$. plantarum ZLP001 at $10^{5}, 10^{6}, 10^{7}, 10^{8}$, or $10^{9} \mathrm{CFU} /$ well for $2 \mathrm{~h}, 3 \mathrm{~h}$, or $4 \mathrm{~h}$. After the bacteria were washed away with PBS, the cells were cultured in a complete culture medium with or without $\mathrm{H}_{2} \mathrm{O}_{2}$ (optimal $\mathrm{H}_{2} \mathrm{O}_{2}$ concentration obtained from the above experiment) for $4 \mathrm{~h}$. Cell viability was determined using the MTT assay.

To estimate the antioxidant effect of $L$. plantarum ZLP001 on IPEC-J2 cells, four treatments were designed: a control, a L. plantarum ZLP001 treatment (optimal concentration and incubation time based on the above experiment), a $\mathrm{H}_{2} \mathrm{O}_{2}$ treatment, and a L. plantarum ZLP001 pretreatment $+\mathrm{H}_{2} \mathrm{O}_{2}$ treatment. After overnight culture, IPEC-J2 cells were incubated with $L$. plantarum ZLP001 in a complete culture medium without antibiotics for $3 \mathrm{~h}$. Then, the bacteria were washed away with PBS, and the cells were cultured in a complete culture medium with or without $\mathrm{H}_{2} \mathrm{O}_{2}$ for $4 \mathrm{~h}$. In addition, IPEC-J2 cells were cultured in a complete culture medium without antibiotics for $3 \mathrm{~h}$ and then treated or not treated with $\mathrm{H}_{2} \mathrm{O}_{2}$ under the conditions described above as oxidative stress control and nontreated control, respectively.

2.4. Observation of Cell Morphology. Morphological changes in IPEC-J2 cells after the treatments were observed under an optical microscope (Olympus, Tokyo, Japan) at a magnification of 100x. Images were acquired using the cellSens Entry system (Olympus).

2.5. Detection of Cell Apoptosis and Necrosis. Apoptosis and necrosis in treated IPEC-J2 cells were detected using the Apoptosis and Necrosis Assay Kit (C1056) (Beyotime Biotechnology, Haimen, China). After the treatments, the cells were stained with Hoechst $33342(10 \mathrm{ng} / \mathrm{mL})$ and propidium iodide (PI, $10 \mathrm{ng} / \mathrm{mL}$ ) at $4^{\circ} \mathrm{C}$ in the dark for $20 \mathrm{~min}$, according to the manufacturer's instructions. Condensed or fragmented nuclei of apoptotic cells were visualized and photographed under an inverted fluorescence microscope (IX71, Olympus). 
Apoptotic cells were also detected by flow cytometry using an Annexin V PE/7-AAD Assay Kit (CA1030) (Solarbio, Beijing, China). Immediately after the treatments, the cells were collected and resuspended in a binding buffer. One hundred microliters of cell suspension were mixed with $5 \mu \mathrm{L}$ of Annexin $\mathrm{V} / \mathrm{PE}$ and incubated in the dark at room temperature for $5 \mathrm{~min}$. After the addition of $10 \mu \mathrm{L}$ of $20 \mu \mathrm{g} / \mathrm{mL} 7-\mathrm{AAD}$ and $400 \mu \mathrm{L}$ of PBS, the cells were immediately analyzed by flow cytometry (FACScaliburTM, BD Biosciences, San Jose, CA, USA). Experiments were performed in triplicate.

2.6. Assessment of Intracellular ROS Generation. Intracellular ROS accumulation was measured using a commercial ROS detection kit (S0033S) (Beyotime Biotechnology, Haimen, China) with the green, fluorescent probe DCFH-DA $\left(2^{\prime}, 7^{\prime}\right.$ -dichlorofluorescein diacetate). After the treatments, the culture medium was removed, the ROS indicator DCFH-DA $(10 \mu \mathrm{M})$ in fresh FBS-free medium was added, and the cells were incubated at $37^{\circ} \mathrm{C}$ for $30 \mathrm{~min}$. The cells were visualized and photographed under a fluorescence microscope (IX71, Olympus). To quantify ROS production, the fluorescence intensity was measured using a fluorescence microplate reader (Tecan, Männedorf, Switzerland) at excitation/emission wavelengths of 525/610 nm. ROS levels are expressed as the percentage of treated cells compared to control cells. Experiments were performed in triplicate.

2.7. Measurement of the Mitochondrial Membrane Potential $(M M P)$. The Mitochondrial Membrane Potential Assay Kit (Beyotime Biotechnology, Haimen, China) with the membrane-permeable dye, JC-1, was used to detect mitochondrial depolarization in cells. After the treatments, the cells were incubated in JC- 1 solution at $37^{\circ} \mathrm{C}$ for $15 \mathrm{~min}$. The potential-dependent aggregation of JC- 1 in the mitochondria (labeled with red fluorescence) and of the monomeric form of JC-1 in the cytosol after mitochondrial membrane depolarization (labeled with green fluorescence) were detected using flow cytometry. The MMP is reflected by the proportion of JC-1 aggregates and monomers. Experiments were performed in triplicate.

2.8. Determination of Lactate Dehydrogenase (LDH) and Malondialdehyde (MDA). After the treatments, the cells were gently washed twice with PBS and lysed using RIPA Lysis Buffer (containing PMSF) (Solarbio, Beijing, China) for $10 \mathrm{~min}$. The cells were centrifuged and $10,000 \times g$ at $4^{\circ} \mathrm{C}$ for $10 \mathrm{~min}$, and the supernatants were collected. Protein concentrations were determined using a bicinchoninic acid (BCA) protein assay (BCA Protein Assay Kit; Pierce, Madison, WI, USA). Then, the levels of $\mathrm{LDH}$ and MDA were determined using a LDH assay kit (A020-2) and MDA assay kit (A0032) (Jiancheng, Nanjing, China). Experiments were performed in triplicate.

2.9. Determination of Glutathione and Oxidized Glutathione (GSH and GSSG) Concentrations. The cell sample collection and protein extraction and concentration evaluation were the same as above for the determination of LDH and MDA. Thereafter, the concentration of GSH and GSSG was deter- mined using a GSH assay kit (A006-1-1) and GSSG assay kit (A061-2-1) (Jiancheng, Nanjing, China). Experiments were performed in triplicate.

2.10. Determination of T-AOC, T-SOD, CAT, and GSH-Px Activities. The total antioxidant capacity (T-AOC, A015-12 ) and the activities of total superoxide dismutase (T-SOD, A001-1-2), catalase (CAT, A007-1-1), and glutathione peroxidase (GSH-Px, A005-1-2) were determined using commercial assay kits (Jiancheng, Nanjing, China). After the treatments, the cells were lysed, and cellular protein concentrations were determined as above. Then, the T-SOD, CAT, and GSH-Px activities are determined according to the instructions of the manufacturer and expressed as U/mg protein. Experiments were performed in triplicate.

2.11. Establishment of Nrf2-Knockdown IPEC-J2 Cells. Porcine Nrf2 siRNA $5^{\prime}$-GCCCAUUGAUCUCUCUGAUTT-3' (sense) and 5' -AUCAGAGAGAUCAAUGGGCTT-3' (antisense) were synthesized at GenePharma Co. Ltd. (Shanghai, China). The IPEC-J2 cells were seeded into 6-well plates $\left(2.5 \times 10^{5}\right.$ cells/well $)$, cultured overnight, and transfected with siRNAs using Lipofectamine 2000 (Invitrogen, Carlsbad, CA, USA) according to the manufacturer's instructions.

2.12. Quantitative Reverse-Transcription Polymerase Chain Reaction (RT-qPCR). Total RNA was extracted from treated cells using RNAzol reagent (Molecular Research Center, Cincinnati, OH, USA) according to the manufacturer's instructions. RNA concentrations were determined using a NanoDrop spectrophotometer (Thermo Fisher Scientific, Waltham, MA, USA). The RNA $(1 \mu \mathrm{g})$ was reversetranscribed into cDNA using an iScript ${ }^{\mathrm{TM}} \mathrm{cDNA}$ Synthesis Kit (Bio-Rad, Hercules, CA, USA) according to the manufacturer's instructions. The qPCRs were run using iTaqTM Universal SYBR Green Supermix (Bio-Rad) in a QuantStudio 3 Real-Time PCR System (Thermo Fisher Scientific, Waltham, MA, USA). Porcine-specific primers used in this study were referred from other references or designed with Primer 5.0, and the sequences are listed in Supplementary Table S1. Target gene expression was normalized to that of glyceraldehyde-3-phosphate dehydrogenase (GAPDH); relative fold changes in gene expression were calculated using the $2^{-\Delta \Delta \mathrm{Ct}}$ method [18].

2.13. Western Blot. IPEC-J2 cells were collected after the treatments, and total proteins were extracted and protein concentrations were determined as above description. Equal amounts of protein $(30 \mu \mathrm{g})$ were loaded per lane and the protein was separated at $110 \mathrm{~V}$ for $1 \mathrm{~h}$ and then transferred to the PDVF membrane at $4^{\circ} \mathrm{C}$ and $90 \mathrm{~V}$ for $60-100 \mathrm{~min}$. After blocking with $5 \%$ skim milk, the blots were incubated with primary antibodies at $4^{\circ} \mathrm{C}$ overnight. After three washes with Tris-buffered saline, the blots were incubated with an HRPconjugated secondary antibody. Chemiluminescence detection was performed using Western Blot Luminance Reagent (Santa Cruz Biotechnology, Santa Cruz, CA, USA) according to the manufacturer's instructions. The antibodies used in this study are listed in Supplementary Table S2. 
Immunoreactive bands were imaged using the ChemiDoc XRS system (Bio-Rad) and were quantified using ImageJ (National Institutes of Health, Bethesda, MD, USA).

2.14. Statistical Analysis. All results are expressed as the mean \pm standard error of the mean (SEM). Data analyses were performed using Prism version 6 (GraphPad Software, Inc., San Diego, CA, USA). Means were compared using one-way analysis of variance (ANOVA) followed by Duncan's post hoc tests in SPSS (version 20.0, SPSS Inc., Chicago, IL, USA). Means of two groups were compared using unpaired Student's two-tailed $t$-test. $P<0.05$ was considered a significant difference.

\section{Results}

3.1. Establishment of an Oxidative Stress Model in IPEC-J2 Cells. To establish the oxidative stress model in porcine IPEC-J2 cells, we used the MTT method to determine the cell viability of IPEC-J2 after treatment with $\mathrm{H}_{2} \mathrm{O}_{2}$. As shown in Figure $1(\mathrm{a}), \mathrm{H}_{2} \mathrm{O}_{2}$ dose-dependently decreased the viability of IPEC-J2 cells. After $4 \mathrm{~h}$ of treatment with $1,000 \mu \mathrm{M}$ $\mathrm{H}_{2} \mathrm{O}_{2}$, the cell viability of IPEC-J2 was reduced to $52.8 \% \pm$ $4.7 \%$. Therefore, this concentration of $1,000 \mu \mathrm{M}$ and $4 \mathrm{~h}$ treatment time were used to induce oxidative stress in subsequent experiments.

3.2. L. plantarum ZLP001 Attenuated the Cell Damage Caused by $\mathrm{H}_{2} \mathrm{O}_{2}$. As shown in Figure 1(b), a 3-h pretreatment with $10^{6}$ or $10^{7} \mathrm{CFU}$ L. plantarum ZLP001 increased IPEC-J2 cell viability by approximately up to $70 \%-75 \%$ after $\mathrm{H}_{2} \mathrm{O}_{2}$ insult, indicating that $L$. plantarum ZLP001 attenuates $\mathrm{H}_{2} \mathrm{O}_{2}$-induced cell damage. Lower and higher concentrations of L. plantarum ZLP001 were less effective, while $10^{9} \mathrm{CFU} L$. plantarum ZLP001 decreased the viability of IPEC-J2 directly and did not protect against $\mathrm{H}_{2} \mathrm{O}_{2}$-induced damage. Pretreatment with L. plantarum ZLP001 for $3 \mathrm{~h}$ more effectively protected cell viability than $2 \mathrm{~h}$ or $4 \mathrm{~h}$ pretreatment. We selected $10^{6} \mathrm{CFU}$ L. plantarum ZLP001 concentration and $3 \mathrm{~h}$ pretreatment time to proceed with further research.

Cell morphology after the different treatments was examined by optical microscopy (Figure 1(c)). Compared with normal cells, the gaps between $\mathrm{H}_{2} \mathrm{O}_{2}$-treated IPEC-J2 cells were enlarged, and the cell membrane showed a loose structure. After pretreatment with L. plantarum ZLP001, less damage to cell integrity was observed.

$\mathrm{LDH}$ is a soluble cytosolic enzyme that is released when the cell membrane is damaged. To confirm the protective effect of L. plantarum ZLP001 on IPEC-J2 cells, LDH leakage after $\mathrm{H}_{2} \mathrm{O}_{2}$ treatment was measured (Figure 1(d)). While $\mathrm{H}_{2} \mathrm{O}_{2}$-treated IPEC-J2 cells showed significant LDH release compared to nontreated cells, pretreatment with L. plantarum ZLP001 strongly reduced LDH leakage in the culture supernatant.

3.3. L. plantarum ZLP001 Alleviates $\mathrm{H}_{2} \mathrm{O}_{2}$-Induced Apoptosis and Necrosis in IPEC-J2 Cells. Hoechst 33342 and PI staining results showed that $\mathrm{H}_{2} \mathrm{O}_{2}$ considerably stimulated apoptosis (bright blue) and necrosis (bright red) in IPEC-J2 cells, indicating $\mathrm{H}_{2} \mathrm{O}_{2}$-induced oxidative damage. L. plantarum
ZLP001 pretreatment significantly ameliorated $\mathrm{H}_{2} \mathrm{O}_{2}$ induced apoptosis and necrosis (Figure 2(a)). These findings were confirmed by flow cytometry results (Figures 2(b) and $2(\mathrm{c}))$.

The expression of the apoptosis-associated proteins, Bcl2 , Bax, and active caspase-3, was evaluated by western blot to further understand the antiapoptotic effect of the L. plantarum ZLP001 strain. Expression of the antiapoptotic factor Bcl-2 was enhanced by L. plantarum ZLP001 treatment, whereas no obvious increase was observed after $\mathrm{H}_{2} \mathrm{O}_{2}$ treatment alone (Figures 2(d) and 2(e)). After $\mathrm{H}_{2} \mathrm{O}_{2}$ treatment, activated caspase- 3 expression was significantly increased, and this induction was alleviated by L. plantarum ZLP001 pretreatment. No significant effect on Bax expression was observed.

3.4. L. plantarum ZLP001 Regulates the Cellular Redox State in $\mathrm{H}_{2} \mathrm{O}_{2}$-Treated IPEC-J2 Cells. To evaluate the regulatory effect of $L$. plantarum ZLP001 on the $\mathrm{H}_{2} \mathrm{O}_{2}$-induced IPEC$\mathrm{J} 2$ cell redox state, we measured intracellular ROS production using a cell-permeable, nonfluorescent probe DCFH-DA (Figures 3(a) and 3(b)). Unsurprisingly, ROS accumulation was significantly increased after exposure to $\mathrm{H}_{2} \mathrm{O}_{2}$, indicating that $\mathrm{H}_{2} \mathrm{O}_{2}$ caused an intracellular burst of ROS in IPEC-J2 cells. However, pretreatment of the cells with L. plantarum ZLP001 obviously suppressed the ROS burst induced by $\mathrm{H}_{2} \mathrm{O}_{2}$ in IPEC-J2 cells.

To further investigate the redox state, the MMP was determined (Figures 3(c) and 3(d)), which can be affected by $\mathrm{H}_{2} \mathrm{O}_{2}$-induced ROS release. After $\mathrm{H}_{2} \mathrm{O}_{2}$ treatment alone, the J-aggregate-to-J-monomer ratio in IPEC-J2 cells was obviously decreased. After L. plantarum ZLP001 pretreatment, this ratio was increased, demonstrating the positive effect of L. plantarum ZLP001 on the redox state in IPECJ2 cells.

Next, we detected GSSG and GSH, which serve as important indicators of the cellular redox state (Figure 3(e)). After $\mathrm{H}_{2} \mathrm{O}_{2}$ treatment, intracellular GSSG levels were significantly increased, while GSH levels were markedly decreased $(P<0.05)$. However, pretreatment with L. plantarum ZLP001 showed a tendency to prevent the effects of $\mathrm{H}_{2} \mathrm{O}_{2}$ on both GSSG $(P>0.05)$ and GSH $(P<0.05)$. The GSSG and GSH levels in L. plantarum ZLP001-treated cells were similar to those in control cells $(P>0.05)$.

MDA is the main product of ROS-induced lipid peroxidation. As an excellent indicator of oxidative stress, we also measured the MDA levels in the treated IPEC-J2 cells. As shown in Figure 3(f), MDA levels in $\mathrm{H}_{2} \mathrm{O}_{2}$-treated IPEC-J2 cells were markedly increased compared to those in control cells, indicating the occurrence of lipid peroxidation. L. plantarum ZLP001 pretreatment significantly inhibited lipid peroxidation in IPEC-J2 cells, as indicated by the lower levels of MDA.

3.5. L. plantarum ZLP001 Upregulates the Antioxidant Defense System in $\mathrm{H}_{2} \mathrm{O}_{2}$-Treated IPEC-J2 Cells. The effect of L. plantarum ZLP001 on the antioxidant defense system in the cells was examined to further investigate how L. plantarum ZLP001 alleviated oxidative stress in the cells. The T- 


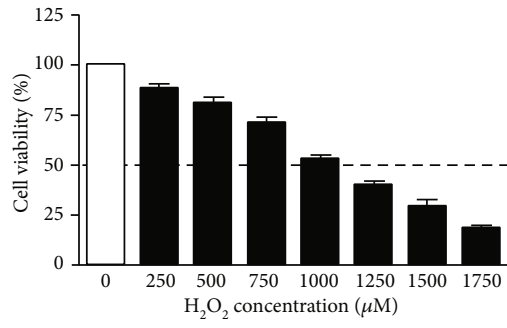

(a)
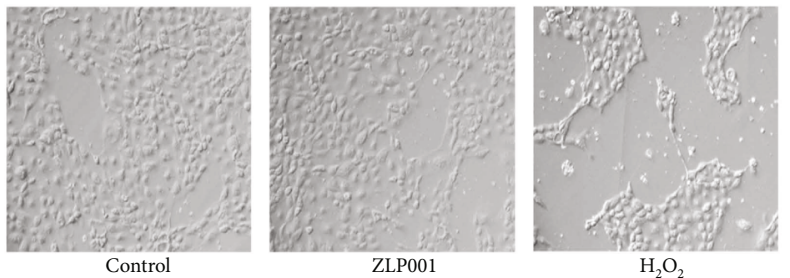

(c)
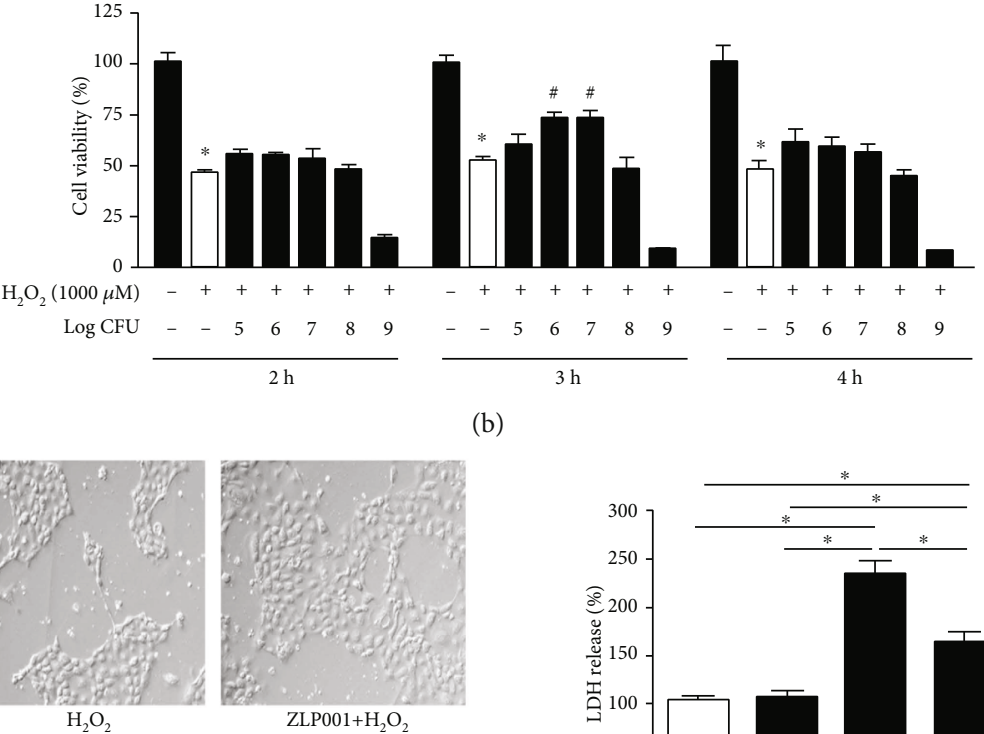

(b)

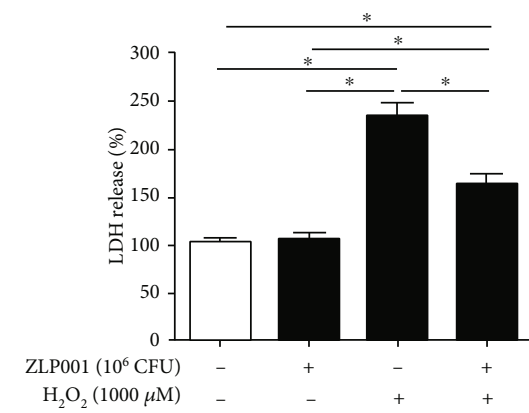

(d)

FIGURE 1: Lactobacillus plantarum ZLP001 protects IPEC-J2 cells against $\mathrm{H}_{2} \mathrm{O}_{2}$-induced oxidative stress damage. (a) $\mathrm{H}_{2} \mathrm{O}_{2}$-induced IPEC-J2 cell viability reduction. IPEC-J2 cells were incubated with the indicated concentrations of $\mathrm{H}_{2} \mathrm{O}_{2}$ for $4 \mathrm{~h}$; after which, cell viability was measured by the methyl thiazolyl tetrazolium (MTT) assay. (b) L. plantarum ZLP001 protects against $\mathrm{H}_{2} \mathrm{O}_{2}$-induced cell damage. IPEC-J2 cells were incubated with or without $L$. plantarum ZLP001 at the indicated concentrations for $2 \mathrm{~h}, 3 \mathrm{~h}$, or $4 \mathrm{~h}$; after which, the medium was replaced with fresh medium containing $1,000 \mu \mathrm{M} \mathrm{H}_{2} \mathrm{O}_{2}$. After incubation for $4 \mathrm{~h}$, cell viability was measured by the MTT assay. (c) Morphological analysis of the protective effect of L. plantarum ZLP001 $\left(10^{6} \mathrm{CFU}, 3 \mathrm{~h}\right)$ against $\mathrm{H}_{2} \mathrm{O}_{2}$-induced IPEC-J2 cell damage by microscopy. (d) Lactate dehydrogenase (LDH) release after the treatments as determined using a LDH assay. All data represent the mean \pm standard error of mean (SEM) of three independent experiments. Means were compared by one-way ANOVA followed by Duncan's post hoc tests. ${ }^{*} P<0.05$. The means of the two groups were compared using Student's $t$-test. ${ }^{*} P<0.05$ vs. nontreated control cells; ${ }^{\#} P<$ 0.05 vs. $\mathrm{H}_{2} \mathrm{O}_{2}$-treated cells.

AOC activities in L. plantarum ZLP001-treated cells showed a numerically increase compared with the normal control group (Figure 4(a)). While the pretreatment of L. plantarum ZLP001 can significantly attenuate the decrease of T-AOC activity caused by $\mathrm{H}_{2} \mathrm{O}_{2}$.

As shown in Figure 4(b), the HO-1, GSTA1, and TRXR1 mRNA expressions were significantly elevated in cell response to $\mathrm{H}_{2} \mathrm{O}_{2}$, whereas $C A T$ expression was significantly decreased, indicating that the cells activated antioxidant mechanisms to protect themselves from oxidative stress. Compared with $\mathrm{H}_{2} \mathrm{O}_{2}$ treatment alone, L. plantarum ZLP001 pretreatment obviously suppressed HO-1 and TRXR1 mRNA expression, whereas it increased SOD1 and CAT expression. L. plantarum ZLP001 treatment alone markedly elevated the mRNA expression of SOD1 and GPX2, whereas no significant effects on other antioxidant enzymes were observed compared with control cells.

We further determined the activities of T-SOD, CAT, and GSH-Px using commercial assay kits (Figures 4(c)$4(\mathrm{e})$ ). In line with the gene expression results, these enzyme activities treated or pretreated with $L$. plantarum ZLP001 were significantly regulated compared with control and $\mathrm{H}_{2} \mathrm{O}_{2}$ treatment alone. After L. plantarum ZLP001 pretreatment, the decline in antioxidant enzymes caused by $\mathrm{H}_{2} \mathrm{O}_{2}$ was alleviated. These results indicate that L. plantarum ZLP001 boosts endogenous antioxidant molecules. Collectively, the antioxidant effect of $L$. plantarum ZLP001 can be attributed to the activation of the antioxidant defense system.

3.6. L. plantarum ZLP001 Activates Nrf2 Signaling Pathway in IPEC-J2 Cells. To further explore the mode of action of L. plantarum ZLP001, we evaluated Nrf2 expression in IPEC-J2 cells treated with different concentrations of L. plantarum ZLP001 (Figures 5(a) and 5(b)). Immunoblotting results revealed that $L$. plantarum ZLP001 treatment dosedependently decreased cytosolic Nrf2 and promoted nuclear Nrf2 accumulation, especially at $10^{7} \mathrm{CFU}$, indicating the occurrence of nuclear translocation of Nrf2. Cytosolic Keap1 accumulation was enhanced after $L$. plantarum ZLP001 treatment, especially at $10^{6}$ and $10^{7} \mathrm{CFU}$.

Next, we evaluated the translocation of Nrf2 after $\mathrm{H}_{2} \mathrm{O}_{2}$ treatment and pretreatment with L. plantarum ZLP001. As shown in Figures $5(\mathrm{c})$ and $5(\mathrm{~d}), \mathrm{H}_{2} \mathrm{O}_{2}$ treatment alone had a remarkable effect on Nrf2 translocation from the cytosol to the nucleus. However, after pretreatment with L. plantarum ZLP001, the amount of Nrf2 in the cytosol showed a further decrease, while no significant accumulation of Nrf2 was observed in the nucleus compared with $\mathrm{H}_{2} \mathrm{O}_{2}$ treatment 


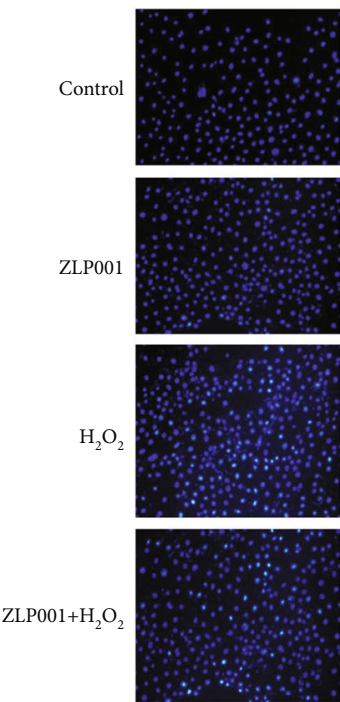

Hoechst 33342

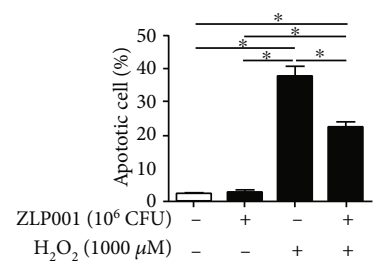

(c)
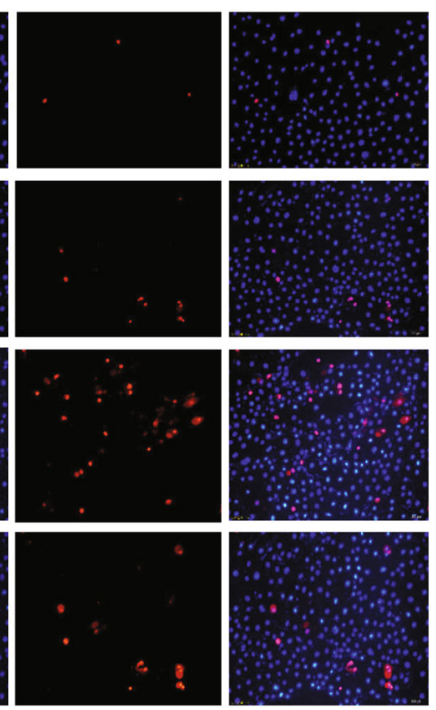

PI

(a)

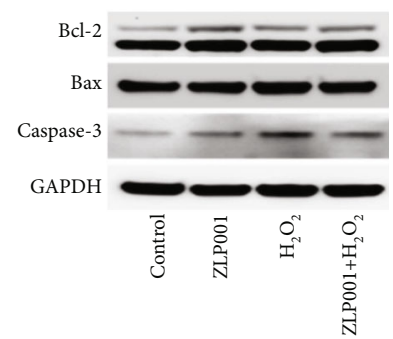

(d)

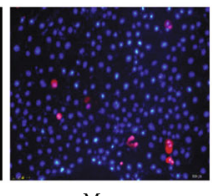

Merge
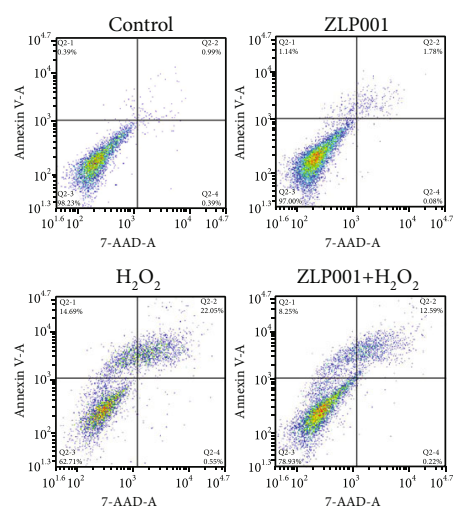

(b)

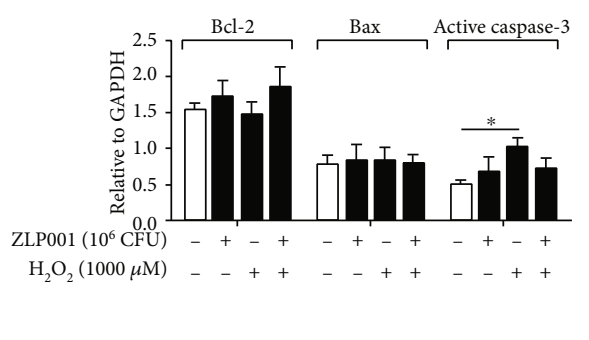

(e)

FIGURE 2: Lactobacillus plantarum ZLP001 inhibits $\mathrm{H}_{2} \mathrm{O}_{2}$-induced apoptosis in IPEC-J2 cells. IPEC-J2 cells were incubated with or without $10^{6} \mathrm{CFU}$ L. plantarum ZLP001 for $3 \mathrm{~h}$; after which, the medium was replaced with a fresh medium containing $1,000 \mu \mathrm{M} \mathrm{H} \mathrm{O}_{2}$, and the cells were further incubated for $4 \mathrm{~h}$. (a) Apoptosis detection based on Hoechst 33342 staining and PI staining. (b) Flow-cytometric analysis of apoptotic cells. (c) Quantification of apoptotic cells based on flow cytometry data. (d) Western blot analysis of Bcl-2, Bax, and active caspase-3 expression. (e) Quantitative analysis of apoptosis-related protein levels. All data represent the mean \pm standard error of mean ( SEM) of three independent experiments. Means were compared by one-way ANOVA followed by Duncan's post hoc test. * $P<0.05$.

alone. The amount of Keap1 in the cytosol numerically increased after L. plantarum ZLP001 treatment alone, and significant differences were observed after $\mathrm{H}_{2} \mathrm{O}_{2}$ treatment alone and pretreatment with L. plantarum ZLP001.

3.7. Nrf2 siRNA Abolishes the Antioxidative Effects of $L$. plantarum ZLP001. To further clarify the role of Nrf2 in the alleviation of oxidative stress by L. plantarum ZLP001, we evaluated cell viability and ROS production after Nrf2 knockdown in IPEC-J2 cells. Cells treated with Nrf2 siRNA effectively exhibited lower levels of Nrf2 than negative control siRNA-transfected cells and nontransfected control cells at both the gene and the protein level (Figures 6(a) and 6(b)). Nrf2 siRNA-transfected IPEC-J2 cells showed considerably lower cell viability than negative control cells when challenged with $\mathrm{H}_{2} \mathrm{O}_{2}$ (Figure 6(c)). The antioxidant-protective effect of $L$. plantarum ZLP001 was also blocked by Nrf2 siRNA, and the cells showed reduced viability when compared with negative control siRNA-transfected cells. Furthermore, Nrf2 knockdown weakened the effect of $L$. plantarum ZLP001 in preventing ROS generation in response to the $\mathrm{H}_{2} \mathrm{O}_{2}$ challenge (Figure 6(d)). These results demonstrate that
Nrf2 siRNA abolishes the antioxidative action of L. plantarum ZLP001, suggesting that the Nrf2 pathway is involved in this action.

\section{Discussion}

An imbalance in the intestinal microbiota under stress or pathological conditions can result in the growth of pathogens, which may produce oxygen to generate an aerobic environment, thus rendering the intestine in an oxidative stress state $[19,20]$. Intestinal oxidative stress causes damage to the epithelial barrier and affects nutrient digestibility and absorption and can lead to various diseases $[3,21,22]$. Thus, intestinal redox homeostasis is critical for maintaining host health. Although some probiotics have antioxidant capacity, the underlying mechanisms are not completely understood. In this study, we aimed to clarify the antioxidant capacity of $L$. plantarum ZLP001 under oxidative stress and its potential mechanism.

$\mathrm{H}_{2} \mathrm{O}_{2}$ is a strong oxidant capable of oxidizing a variety of moieties, which is why it is commonly used to establish an oxidative stress model to study redox-regulated processes in 


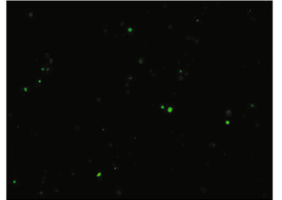

Control

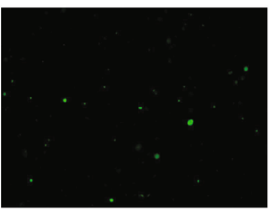

ZLP001

(a)
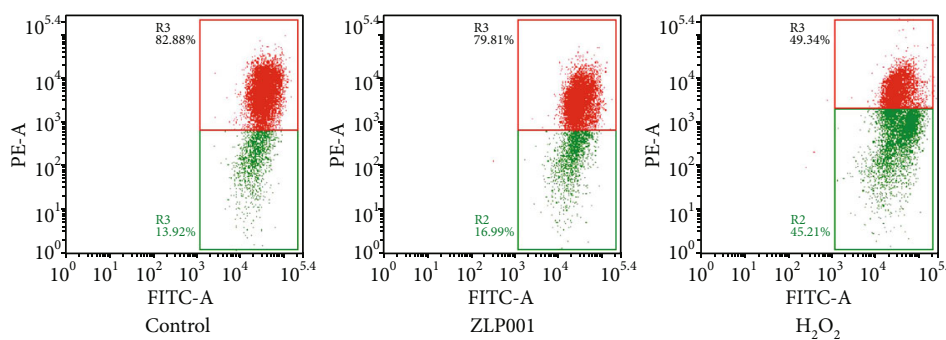

(c)

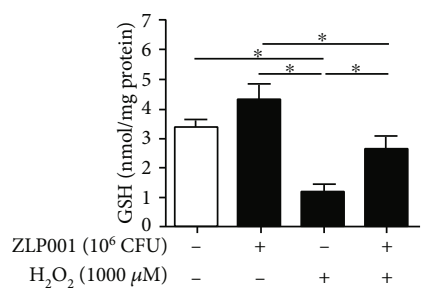

(e)

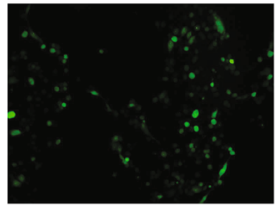

$\mathrm{ZLP001}+\mathrm{H}_{2} \mathrm{O}_{2}$

$\mathrm{H}_{2} \mathrm{O}_{2}$

$\mathrm{H}_{2} \mathrm{O}_{2}$

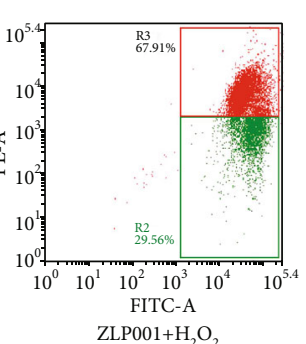

$\begin{array}{rrrrr}\mathrm{ZLP} 001\left(10^{6} \mathrm{CFU}\right) & - & + & - & + \\ \mathrm{H}_{2} \mathrm{O}_{2}(1000 \mu \mathrm{M}) & - & - & + & +\end{array}$

(d)

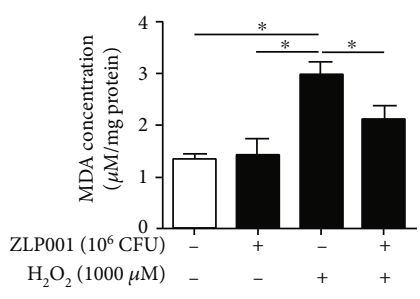

(f)

FIgURE 3: Lactobacillus plantarum ZLP001 improves the $\mathrm{H}_{2} \mathrm{O}_{2}$-induced redox state in IPEC-J2 cells. IPEC-J2 cells were incubated with or without $10^{6} \mathrm{CFU}$ L. plantarum ZLP001 for $3 \mathrm{~h}$; after which, the medium was replaced with a fresh medium containing $1,000 \mu \mathrm{M} \mathrm{H} \mathrm{H}_{2}$, and the cells were further incubated for $4 \mathrm{~h}$. (a) L. plantarum ZLP001 prevents $\mathrm{H}_{2} \mathrm{O}_{2}$-induced ROS accumulation in IPEC-J2 cells as indicated by DCFH-DA staining. (b) Quantification of the ROS levels based on DCFH-DA fluorescence. (c) Effect of L. plantarum ZLP001 on the mitochondrial membrane potential (MMP) in $\mathrm{H}_{2} \mathrm{O}_{2}$-induced IPEC-J2 cells as analyzed by flow cytometry. (d) Quantification of the effect on the MMP based on flow-cytometric data. (e) Concentrations of glutathione (GSH) and oxidized glutathione (GSSG) were determined using GSSG and GSH detection assays. (f) Concentrations of malondialdehyde (MDA) were determined using an MDA assay kit. All data represent the mean \pm standard error of mean (SEM) of three independent experiments. Means were compared one-way ANOVA followed by Duncan's post hoc tests. ${ }^{*} P<0.05$.

various cell types [23-25]. In the present study, MTT assays and the LD50 results demonstrated that treatment with $1,000 \mu \mathrm{M} \mathrm{H}_{2} \mathrm{O}_{2}$ for $4 \mathrm{~h}$ was sufficient to induce oxidative stress in IPEC-2 cells. Pretreatment with L. plantarum ZLP001 $\left(10^{6} \mathrm{CFU}, 3 \mathrm{~h}\right)$ significantly alleviated the decrease of cell viability and release of $\mathrm{LDH}$ caused by $\mathrm{H}_{2} \mathrm{O}_{2}$, suggesting that L. plantarum ZLP001 has antioxidant capacity and can protect cell integrity. Previous studies have demonstrated that epithelial barrier injury is associated with oxidative stress $[22,26]$. Our previous study revealed that L. plantarum ZLP001 has a positive effect on intestinal barrier function [27]; therefore, we speculate that the fortifying effect of $L$. plantarum ZLP001 on the intestinal barrier may be partially associated with its antioxidant ability. We used morphological analysis to observe nuclear condensation and DNA fragmentation, which are hallmarks of cell apoptosis [28, 29]. Apoptosis is generally induced when cells are subjected to oxidative stress $[30,31] . \mathrm{H}_{2} \mathrm{O}_{2}$-treated IPEC-J2 cells showed obvious apoptosis, as demonstrated by the appearance of apoptotic nuclei based on Hoechst staining, compared with control cells. Pretreatment with L. plantarum ZLP001 obviously lowered the population of apoptotic nuclei in $\mathrm{H}_{2} \mathrm{O}_{2}$ induced IPEC-J2 cells. Flow cytometry results confirmed these findings. Probiotics have been previously demonstrated to have antiapoptotic effects and thus improve barrier function [32], suggesting they can indeed improve cell viability. Further, treatment with L. plantarum ZLP001 enhanced the expression of $\mathrm{Bcl}-2$, while pretreatment suppressed the increase in activated caspase- 3 caused by $\mathrm{H}_{2} \mathrm{O}_{2}$, corroborating the potential antiapoptotic role of $L$. plantarum ZLP001. Similar results were obtained by [14] using the probiotic strain Bacillus amyloliquefaciens SC06, although they only detected apoptosis-related gene expression and showed the image results. Taken together, our results indicate that L. plantarum ZLP001 has the potential to preserve intestinal integrity and barrier function under oxidative stress.

To confirm the antioxidant effect of $L$. plantarum ZLP001, we determined ROS production, MDA levels, and MMP. ROS are generated essentially for cellular growth and proliferation and have regulatory effects under 


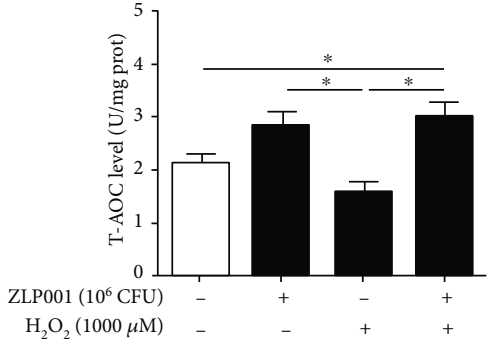

(a)

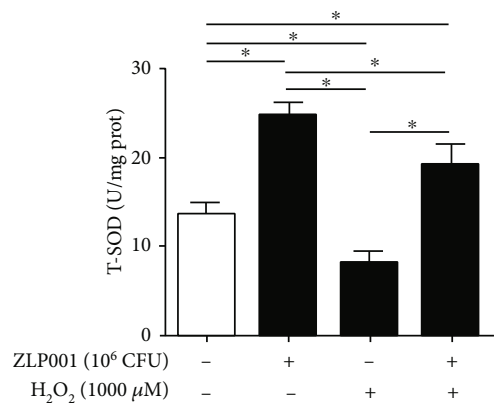

(c)

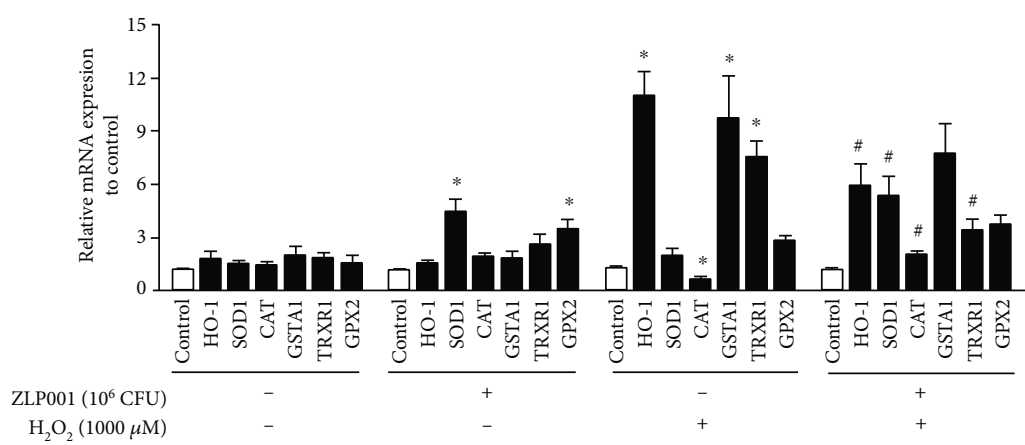

(b)

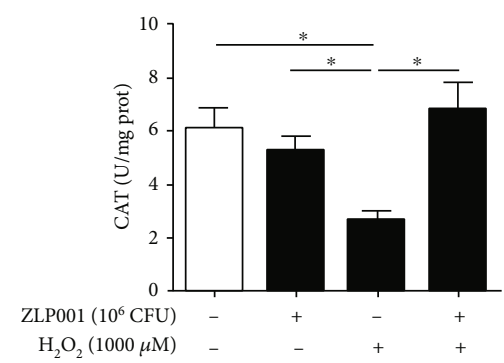

(d)

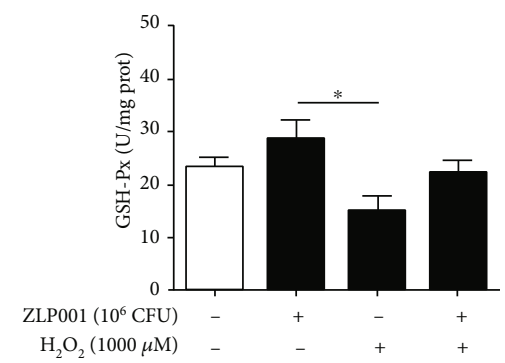

(e)

FIGURE 4: Effect of Lactobacillus plantarum ZLP001 on antioxidant enzyme expression and activity in IPEC-J2 cells. IPEC-J2 cells were incubated with $10^{6} \mathrm{CFU}$ L. plantarum ZLP001 for $3 \mathrm{~h}$; after which, the medium was replaced with a fresh medium containing $1,000 \mu \mathrm{M}$ $\mathrm{H}_{2} \mathrm{O}_{2}$, and the cells were further incubated for $4 \mathrm{~h}$. (a) T-SOD activity in lysed cells was determined using a commercial assay kit. (b) The mRNA levels of HO-1, SOD-1, CAT, GSTA1, TRXR1, and GPX2 as determined by RT-qPCR. (c-e) T-SOD, CAT, GSH-Px activities in lysed cells were determined using commercial assay kits. All data represent the mean \pm standard error of mean (SEM) of three independent experiments. Differences between groups were analyzed by one-way ANOVA followed by Duncan's post hoc tests. ${ }^{*} P<0.05$. Differences between two groups were determined by Student's $t$-test. ${ }^{*} P<0.05$ vs. nontreated control cells; ${ }^{\#} P<0.05$ vs. $\mathrm{H}_{2} \mathrm{O}_{2}$-treated cells.

physiological conditions [33-35]. Under normal conditions, intracellular ROS levels are maintained at a sustainable level. However, ROS overproduction, which occurs when ROS levels exceed the endogenous cellular capacity to remove them, will lead to oxidative damage of intracellular macromolecules, thus inducing a series of oxidative stress reactions [36]. $\mathrm{H}_{2} \mathrm{O}_{2}$ induced an intracellular ROS burst in IPEC-J2 cells as revealed by DCFH-DA staining. Pretreatment with L. plantarum ZLP001 remarkably reduced ROS accumulation induced by $\mathrm{H}_{2} \mathrm{O}_{2}$. The conversion of GSH to GSSG corroborated that L. plantarum ZLP001 could alleviate the oxidative stress caused by $\mathrm{H}_{2} \mathrm{O}_{2}$. GSH actively participates in scavenging ROS, but the conversion of GSH to GSSG after oxidation leads to the protein glutathionylation [37]. Levels of MDA, a product of lipid peroxidation, exhibited similar trends as the ROS levels in the present study. $\mathrm{H}_{2} \mathrm{O}_{2}$ treatment stimulated MDA secretion, whereas L. plantarum ZLP001 pretreatment inhibited the production of MDA induced by $\mathrm{H}_{2} \mathrm{O}_{2}$. As the largest contributors to intracellular oxidant production in most cell types, mitochondria are the site of major oxidative processes [38] and play a critical role in the oxidative stress-induced cell death [39]. We measured the MMP to evaluate the redox state of mitochondria. $\mathrm{H}_{2} \mathrm{O}_{2}$ decreased the MMP, whereas L. plantarum ZLP001 pretreatment enhanced the MMP. Mitochondrial dysfunction as indicated by a decrease in the MMP is considered a characteristic feature of early apoptosis [40]. The decrease in the MMP after $\mathrm{H}_{2} \mathrm{O}_{2}$ treatment in the present study suggested that apoptosis would be induced in the IPEC-J2 cells. Pretreatment with L. plantarum ZLP001 enhanced the MMP and markedly suppressed IPEC-J2 apoptosis. Thus, L. plantarum ZLP001 can effectively modulate the antioxidant status in IPEC-J2 cells. The limitation of our study is that the active molecules of this strain were not deeply studied. Studies have demonstrated that antioxidant molecules produced by probiotic strains, like exopolysaccharides and ferulic acid, may the key factors that probiotics play their antioxidant activity [41, 42]. Further isolation, purification, and structural elucidation may also need to evaluate the main active ingredients of L. plantarum ZLP001.

Enhancing the host antioxidant defense system leads to ROS scavenging in the body, thus alleviating oxidative stress. The protective effect of probiotics involves both nonenzymatic and enzymatic redox mechanisms [43]. To further investigate the antioxidant mechanisms of L. plantarum ZLP001, we evaluated T-AOC and mRNA expression and activities of some antioxidant-associated enzymes in IPEC2 lysates. T-AOC normally reflects the capacity of the nonenzymatic antioxidant defense system [44] and is often used as a biomarker to investigate oxidative status [45]. The elevation 


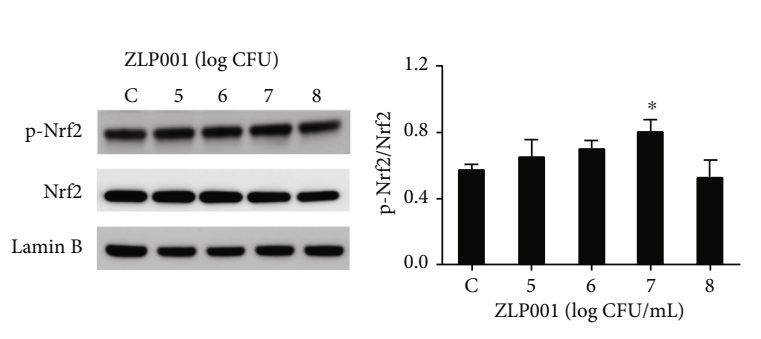

(a)

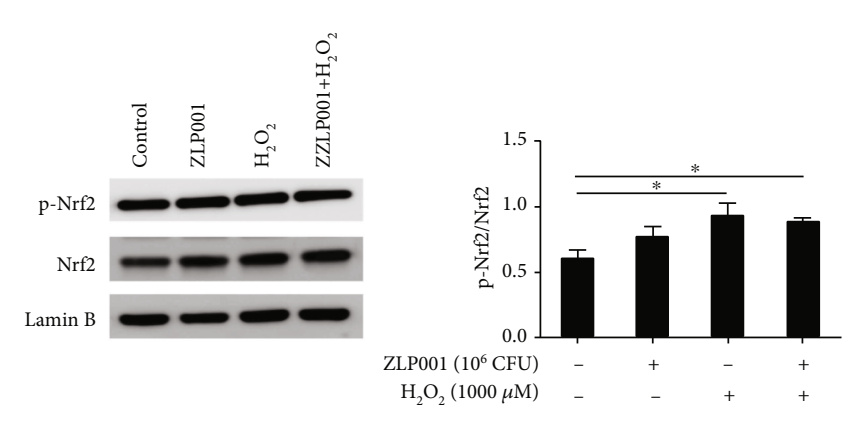

(c)

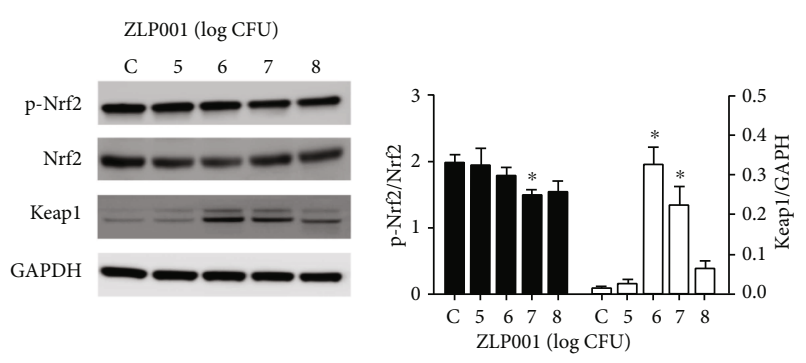

(b)

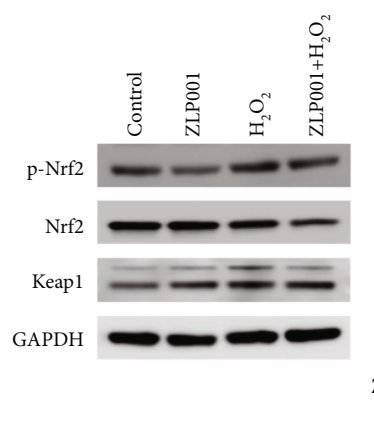

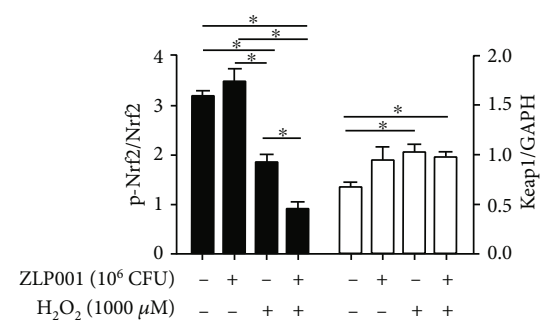

(d)

Figure 5: Effects of Lactobacillus plantarum ZLP001 on the Nrf2/Keap1 pathways in IPEC-J2 cells. (a) Protein levels of Nrf2 and p-Nrf2 as detected by western blot in nucleus (treated with different concentrations of L. plantarum ZLP001). (b) Protein levels of Nrf2, p-Nrf2, and Keap1 as detected by western blot in cytoplasm (treated with different concentrations of L. plantarum ZLP001). IPEC-J2 cells were incubated with indicated concentrations of L. plantarum ZLP001 for $3 \mathrm{~h}$. After isolation of the nuclei from the cells, the nuclear Nrf2 and cytosolic Nrf2 and Keap1 were determined by immunoblotting. (c) Protein levels of Nrf2 and p-Nrf2 as detected by western blot in nucleus (treated with L. plantarum ZLP001 followed by $\mathrm{H}_{2} \mathrm{O}_{2}$ ). (d) Protein levels of Nrf2, p-Nrf2, and Keap1 as detected by western blot in cytoplasm (treated with $L$. plantarum ZLP001 followed by $\mathrm{H}_{2} \mathrm{O}_{2}$ ). IPEC-J2 cells were incubated with or without $10^{6} \mathrm{CFU}$ L. plantarum ZLP001 for $3 \mathrm{~h}$; after which, the medium was replaced with a fresh medium containing $1,000 \mu \mathrm{M} \mathrm{H}_{2} \mathrm{O}_{2}$, and the cells were further incubated for $4 \mathrm{~h}$. After isolation of the nuclei from the cells, the nuclear Nrf2 and cytosolic Nrf2 and Keap1 were determined by immunoblotting. All data represent the mean \pm standard error of mean (SEM) of three independent experiments. Differences between the two groups were determined by Student's $t$-test. ${ }^{*} P<0.05$ vs. nontreated control cells. Differences between groups were analyzed by oneway ANOVA followed by Duncan's post hoc tests. ${ }^{*} P<0.05$.

in T-AOC after pretreatment with L. plantarum ZLP001 demonstrated that L. plantarum ZLP001 suppresses oxidative stress at least in part via the nonenzymatic antioxidant defense system. HO-1, a phase II enzyme, is transcriptionally regulated by various stimuli [46]. HO- 1 is extremely sensitive to $\mathrm{H}_{2} \mathrm{O}_{2}$ induction and can be used as a sensitive target to screen antioxidants [14]. In the present study, we observed induction of HO- 1 gene expression by $\mathrm{H}_{2} \mathrm{O}_{2}$, which was suppressed by L. plantarum ZLP001 pretreatment. Antioxidant enzymes, such as SOD and CAT, can detoxify ROS to safe molecules, thus protecting cells against ROS damage [47, 48]. Increased expression of SOD1 after L. plantarum ZLP001 treatment alone and increased CAT expression after L. plantarum ZLP001 pretreatment compared with $\mathrm{H}_{2} \mathrm{O}_{2}$ treatment was observed in the present study. Therefore, we also determined the expression levels of some other antioxidant-associated genes. L. plantarum ZLP001 treatment alone increased GPX2 expression to elevate the antioxidant capacity. Similar results have been obtained in Labeo rohita fed probiotic and symbiotic diets [49]. The increase in GSTA1 expression after $\mathrm{H}_{2} \mathrm{O}_{2}$ exposure may suggest a self-protective response to mitigate $\mathrm{H}_{2} \mathrm{O}_{2}$ toxicity [50]. Thioredoxin system activation was also observed after
$\mathrm{H}_{2} \mathrm{O}_{2}$ treatment, and L. plantarum ZLP001 pretreatment decreased TRXR1 expression, which is consistent with findings for several Bacillus strains research [14].

To confirm that L. plantarum ZLP001 enhanced the antioxidant system in IPEC-J2 cells, we measured antioxidase activity in IPEC-J2 cell lysates. In line with the gene expression results, L. plantarum ZLP001 increased T-SOD activity, and L. plantarum ZLP001 pretreatment alleviated the suppression of these enzyme activities by $\mathrm{H}_{2} \mathrm{O}_{2}$. The regulatory effects of probiotic strains on host antioxidative enzymes have been widely verified in vitro (in various cell models) and in vivo (in serum and in diverse tissues) [43, 50-52]. Our findings suggest that L. plantarum ZLP001 can dramatically improve the antioxidant status in IPEC-J2 cells by promoting cellular antioxidant defense systems against species generating ROS, thus rendering cells more resistant to the $\mathrm{H}_{2} \mathrm{O}_{2}$ challenge.

As the key endogenous pathway regulating the antioxidant system, activation of the Nrf2/Keap1-antioxidant response element (ARE) axis increases the transcription of antioxidant response elements, thus protecting cells and tissues against ROS damage [53-55]. The altered phase II gene expression and antioxidase activities imply that L. plantarum 


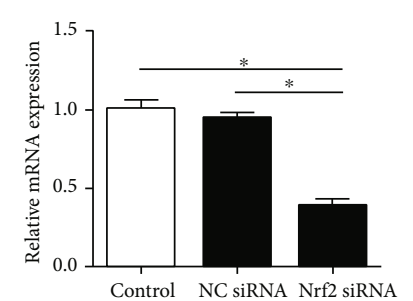

(a)

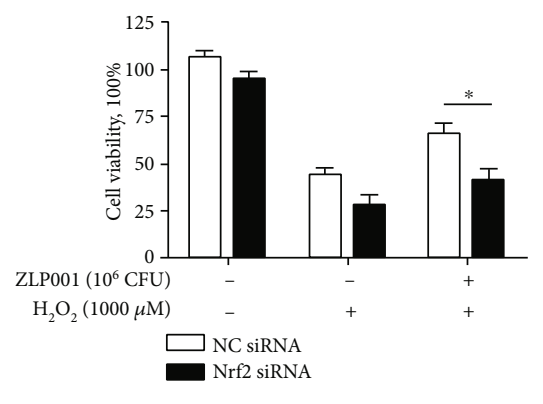

(c)

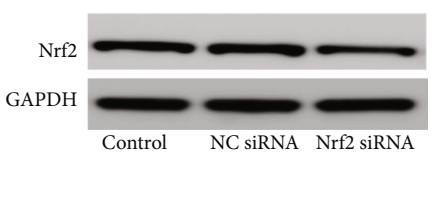

(b)

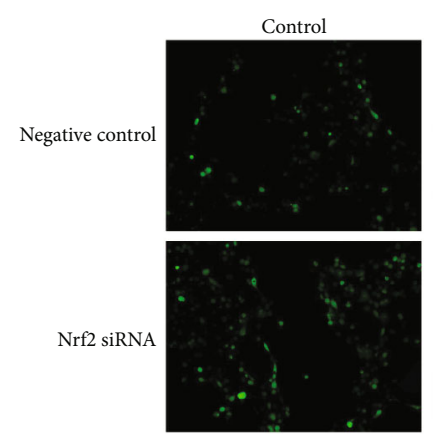

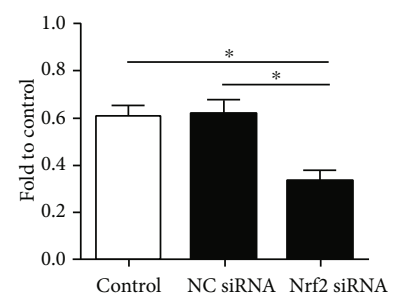
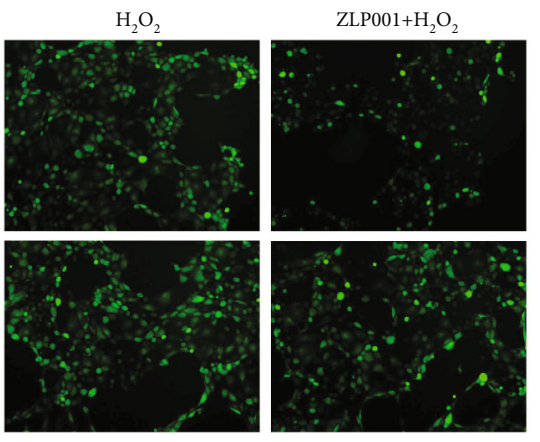

(d)

FIGURE 6: Effect of Lactobacillus plantarum ZLP001 on $\mathrm{H}_{2} \mathrm{O}_{2}$-induced oxidant stress in Nrf2-knockdown IPEC-J2 cells. IPEC-J2 cells were transfected with Nrf2 siRNA or negative control siRNA, and the efficiency of Nrf2 silencing was evaluated by RT-qPCR and western blot. (a) The mRNA level of Nrf2. (b) Western blot analysis of Nrf2 protein levels. Nrf2-knockdown and control cells were incubated with or without $10^{6} \mathrm{CFU}$ L. plantarum ZLP001 for $3 \mathrm{~h}$; after which, the medium was replaced with a fresh medium containing $1,000 \mu \mathrm{M} \mathrm{H}_{2} \mathrm{O}_{2}$, and the cells were further incubated for $4 \mathrm{~h}$. (c) Cell viability was measured using the methyl thiazolyl tetrazolium assay. The results were normalized to negative control siRNA-transfected cells without $\mathrm{H}_{2} \mathrm{O}_{2}$ and $L$. plantarum ZLP001 treatment. (d) ROS production was assessed by DCFH-DA staining and fluorescence microscopy. All data represent the mean \pm standard error of mean (SEM) of three independent experiments. Differences between groups were analyzed by one-way ANOVA followed by Duncan's post hoc tests. ${ }^{*} P<0.05$. Differences between the two groups were determined using Student's $t$ test. ${ }^{*} P<0.05$ vs. negative control siRNA-treated cells.

ZLP001 may affect the Nrf2/Keap1 pathway. Therefore, we investigated whether the Nrf2/Keap1-ARE pathway was involved in the antioxidant function of $L$. plantarum ZLP001. The expression of Nrf2/Keap1 pathway-related proteins in IPEC-J2 cells was enhanced by L. plantarum ZLP001, although differences were only significant at $10^{7} \mathrm{CFU}$. Previous studies have also revealed that probiotic administration in high fat diet-fed mice can induce Nrf2 expression compared with the control, and probiotic treatment alone in vitro was able to induce Nrf2 phosphorylation in IPEC-1 cells $[14,56]$. However, as different probiotic strains induce variable levels of Nrf2 expression [57], we plan to conduct a comparative study of multiple strains and to investigate other potential pathways. Nrf2 is normally activated when cells are exposed to oxidants and electrophiles, thus protecting the cells against oxidative stress [54]. $\mathrm{H}_{2} \mathrm{O}_{2}$ stress increased Nrf2 phosphorylation in the present study, which concurs with Wang et al. [14]. After pretreatment with L. plantarum ZLP001, we observed enhanced dissociation of Nrf2 in the cytosol, but it did not accumulate in the nucleus compared to $\mathrm{H}_{2} \mathrm{O}_{2}$ treatment alone. Kobayashi and Yamamoto [58] reported that a phase II-activated defense system makes cells more resistant to subsequent even greater challenges. Thus, it is possible that L. plantarum ZLP001 may have activated Nrf2 before our detection time point, which made the IPEC-J2 cells more resistant to the subsequent challenge with $\mathrm{H}_{2} \mathrm{O}_{2}$ without a need for the continued accumulation of
Nrf2. We further evaluated the cell viability, ROS production, and mRNA expression levels of antioxidative enzymes after Nrf2 knockdown in IPEC-J2 cells. The protective effect of $L$. plantarum ZLP001 on $\mathrm{H}_{2} \mathrm{O}_{2}$-induced IPEC-J2 cell damage was abolished under Nrf2 deficiency. These results confirm that the Nrf2-ARE signaling pathway is involved in the protective effect of L. plantarum ZLP001 and regulates the expression and activity of antioxidative enzymes to strengthen the defense against $\mathrm{H}_{2} \mathrm{O}_{2}$-induced oxidative stress in IPEC-J2 cells. Similarly, previous studies in different cells and hosts have reported that probiotic strains attenuate oxidative stress by upregulating Nrf2 expression, and by increasing the expression of antioxidative and cytoprotective genes [14, 15, 59]. However, Nrf2 activation by L. plantarum ZLP001 was not as significant as we expected and was not consistent with the antioxidant activity. As we have mentioned, other signaling pathways may also be involved in antioxidant regulation of L. plantarum ZLP001. Probiotics can attenuate hepatic oxidative stress via activating SIRT1 signaling [60] and ameliorate $\mathrm{H}_{2} \mathrm{O}_{2}$-induced epithelial barrier disruption through a PKC- and MAPK-dependent mechanism [61]. Whether these pathways were contributed to the antioxidant activity of $L$. plantarum ZLP001 is unknown. Thus, more robust, in-depth studies are required to unravel the precise antioxidant mechanism of L. plantarum ZLP001. 


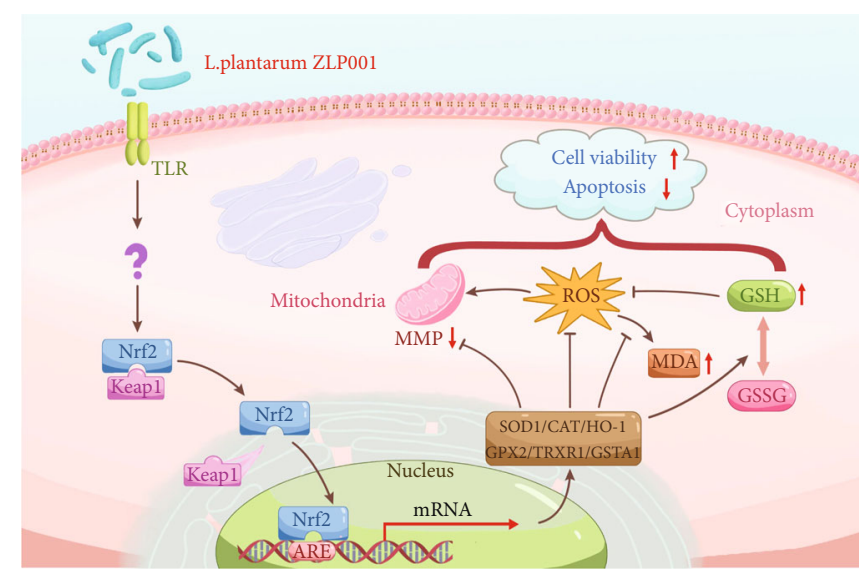

Figure 7: Suggested antioxidant protection mechanism of Lactobacillus plantarum ZLP001. L. plantarum ZLP001 may activate the Keap1-Nrf2 complex; after which, dissociated Nrf2 translocates to the nucleus and activates the transcription of cytoprotective genes, regulating the cellular redox state and activating the antioxidant defense system, thus alleviating cell apoptosis and necrosis caused by $\mathrm{H}_{2} \mathrm{O}_{2}$-induced oxidative stress.

\section{Conclusions}

We demonstrated that L. plantarum ZLP001 can alleviate oxidative damage induced by $\mathrm{H}_{2} \mathrm{O}_{2}$ in porcine epithelial cells. Pretreatment with $L$. plantarum ZLP001 alleviated $\mathrm{H}_{2} \mathrm{O}_{2}$ induced cell oxidative damage by regulating the redox state of cells and enhancing the antioxidant defense system, and Nrf2/Keap1-ARE signaling is involved in its protective mechanisms (Figure 7). Our results improve our understanding of the underlying mechanism of L. plantarum ZLP001 in protecting against oxidative stress, and it can be developed into a therapeutic or protective treatment for animals under oxidative stress. However, whether Nrf2 signaling is activated at an earlier time point as we speculated and which signaling triggered the dissociation of Nrf2 from its constitutive inhibitor, Keap1, or other potential pathways contribute to its antioxidation remain to be investigated more extensively.

\section{Data Availability}

The data generated during the present study are available from the corresponding authors upon request.

\section{Conflicts of Interest}

The authors declare no conflict of interest.

\section{Authors' Contributions}

JW conceived the study and drafted the manuscript. JW, WZ, and $\mathrm{XCH}$ performed the experiments. $\mathrm{WZ}$ analyzed the data. SW and YW contributed to the formal analysis. JW and HJ edited the manuscript. JW and WZ contributed equally to this work.

\section{Acknowledgments}

The authors would like to thank Dr. Ri Qi for helpful suggestions and Dr. Tao Feng for strong support. This study was supported by the Beijing Natural Science Foundation (grant number 6202004), the National Natural Science Foundation of China (grant number 31972576), and the Special Program on Science and Technology Innovation Capacity Building of BAAFS (grant number KJCX201914).

\section{Supplementary Materials}

Supplementary Table S1: sequences of the primers used in this study. Supplementary Table S2: antibodies used in this study. (Supplementary Materials)

\section{References}

[1] M. Schieber and N. S. Chandel, "ROS function in redox signaling and oxidative stress," Current Biology, vol. 24, no. 10, pp. R453-R462, 2014.

[2] J. Lykkesfeldt and O. Svendsen, "Oxidants and antioxidants in disease: oxidative stress in farm animals," Veterinary Journal, vol. 173, no. 3, pp. 502-511, 2007.

[3] J. X. Zhang, L. Y. Guo, L. Feng et al., "Soybean $\beta$-conglycinin induces inflammation and oxidation and causes dysfunction of intestinal digestion and absorption in fish," PLoS One, vol. 8, no. 3, article e58115, 2013.

[4] A. Bhattacharyya, R. Chattopadhyay, S. Mitra, and S. E. Crowe, "Oxidative stress: an essential factor in the pathogenesis of gastrointestinal mucosal diseases," Physiological Reviews, vol. 94, no. 2, pp. 329-354, 2014.

[5] A. Salami, A. Guinguina, J. O. Agboola, A. A. Omede, E. M. Agbonlahor, and U. Tayyab, "Review: In vivo and postmortem effects of feed antioxidants in livestock: a review of the implications on authorization of antioxidant feed additives," Animal, vol. 10, no. 8, pp. 1375-1390, 2016.

[6] S. Janciauskiene, "The beneficial effects of antioxidants in health and diseases," Chronic Obstructive Pulmonary Diseases, vol. 7, no. 3, pp. 182-202, 2020.

[7] V. Mishra, C. Shah, N. Mokashe, R. Chavan, H. Yadav, and J. Prajapati, "Probiotics as potential antioxidants: a systematic review," Journal of Agricultural and Food Chemistry, vol. 63, no. 14, pp. 3615-3626, 2015.

[8] Y. Wang, Y. Wu, Y. Wang et al., "Antioxidant properties of probiotic bacteria," Nutrients, vol. 9, no. 5, p. 521, 2017.

[9] T. Feng and J. Wang, "Oxidative stress tolerance and antioxidant capacity of lactic acid bacteria as probiotic: a systematic review," Gut Microbes, vol. 12, no. 1, article 1801944, 2020.

[10] M. Y. Lin and C. L. Yen, "Antioxidative ability of lactic acid bacteria," Journal of Agricultural and Food Chemistry, vol. 47, no. 4, pp. 1460-1466, 1999.

[11] T. Virtanen, A. Pihlanto, S. Akkanen, and H. Korhonen, "Development of antioxidant activity in milk whey during fermentation with lactic acid bacteria," Journal of Applied Microbiology, vol. 102, no. 1, pp. 106-115, 2007.

[12] G. Mu, Y. Gao, Y. Tuo et al., "Assessing and comparing antioxidant activities of lactobacilli strains by using different chemical and cellular antioxidant methods," Journal of Dairy Science, vol. 101, no. 12, pp. 10792-10806, 2018. 
[13] A. Amaretti, M. di Nunzio, A. Pompei, S. Raimondi, M. Rossi, and A. Bordoni, "Antioxidant properties of potentially probiotic bacteria: in vitro and in vivo activities," Applied Microbiology and Biotechnology, vol. 97, no. 2, pp. 809-817, 2013.

[14] Y. Wang, Y. Wu, Y. Wang et al., "Bacillus amyloliquefaciens SC06 alleviates the oxidative stress of IPEC-1 via modulating Nrf2/Keap1 signaling pathway and decreasing ROS production," Applied Microbiology and Biotechnology, vol. 101, no. 7, pp. 3015-3026, 2017.

[15] X. Lin, Y. Xia, G. Wang et al., "Lactobacillus plantarum AR501 alleviates the oxidative stress of d-galactose-induced aging mice liver by upregulation of Nrf2-mediated antioxidant enzyme expression," Journal of Food Science, vol. 83, no. 7, pp. 1990-1998, 2018.

[16] J. Wang, H. F. Ji, D. Y. Zhang et al., "Assessment of probiotic properties of Lactobacillus plantarum ZLP001 isolated from gastrointestinal tract of weaning pigs," African Journal of Biotechnology, vol. 10, no. 54, pp. 11303-11308, 2011.

[17] J. Wang, H. Ji, S. Wang et al., "Lactobacillus plantarum ZLP001: in vitro assessment of antioxidant capacity and effect on growth performance and antioxidant status in weaning piglets," Asian-Australasian Journal of Animal Sciences, vol. 25, no. 8, pp. 1153-1158, 2012.

[18] K. J. Livak and T. D. Schmittgen, "Analysis of relative gene expression data using real-time quantitative PCR and the $2^{-\Delta \Delta C}$ method," Methods, vol. 25, no. 4, pp. 402-408, 2001.

[19] L. Y. Hu, D. W. Chen, P. Zheng et al., "The bidirectional interactions between resveratrol and gut microbiota: an insight into oxidative stress and inflammatory bowel disease therapy," Biomed Research International, vol. 2019, Article ID 5403761, 9 pages, 2019.

[20] B. Mishra and R. Jha, "Oxidative stress in the poultry gut: potential challenges and interventions," Frontiers in Veterinary Science, vol. 6, p. 60, 2019.

[21] A. Thomson, D. Hemphill, and K. N. Jeejeebhoy, "Oxidative stress and antioxidants in intestinal disease," Journal of Digestive Diseases, vol. 16, no. 3, pp. 152-158, 1998.

[22] Z. Sun, K. Olanders, Å. Lasson et al., "Effective treatment of gut barrier dysfunction using an antioxidant, a PAF inhibitor, and monoclonal antibodies against the adhesion molecule PECAM-1," Journal of Surgical Research, vol. 105, no. 2, pp. 220-233, 2002.

[23] Y. Zou, J. Wang, J. Peng, and H. Wei, “Oregano essential oil induces SOD1 and GSH expression through Nrf2 activation and alleviates hydrogen peroxide-induced oxidative damage in IPEC-J2 cells," Oxidative Medicine and Cellular Longevity, vol. 2016, Article ID 5987183, 13 pages, 2016.

[24] X. Chi, X. Ma, Z. Li et al., "Protective effect of epigallocatechin3 -gallate in hydrogen peroxide-induced oxidative damage in chicken lymphocytes," Oxidative Medicine and Cellular Longevity, vol. 2020, Article ID 7386239, 15 pages, 2020.

[25] J. J. Yang, X. B. Song, Y. Feng et al., "Natural ingredientsderived antioxidants attenuate $\mathrm{H}_{2} \mathrm{O}_{2}$-induced oxidative stress and have chondroprotective effects on human osteoarthritic chondrocytes via Keap1/Nrf2 pathway," Free Radical Biology and Medicine, vol. 152, pp. 854-864, 2020.

[26] I. E. Blasig, C. Bellmann, J. Cording et al., "Occludin protein family: oxidative stress and reducing conditions," Antioxidants \& Redox Signaling, vol. 15, no. 5, pp. 1195-1219, 2011.

[27] J. Wang, H. F. Ji, S. X. Wang et al., "Probiotic Lactobacillus plantarum promotes intestinal barrier function by strengthen- ing the epithelium and modulating gut microbiota," Frontiers in Microbiology, vol. 9, p. 1953, 2018.

[28] Y. Higuchi, "Chromosomal DNA fragmentation in apoptosis and necrosis induced by oxidative stress," Biochemical Pharmacology, vol. 66, no. 8, pp. 1527-1535, 2003.

[29] E. A. Prokhorova, A. V. Zamaraev, G. S. Kopeina, B. Zhivotovsky, and I. N. Lavrik, "Role of the nucleus in apoptosis: signaling and execution," Cellular and Molecular Life Sciences, vol. 72, no. 23, pp. 4593-4612, 2015.

[30] K. Sinha, J. Das, P. B. Pal, and P. C. Sil, “Oxidative stress: the mitochondria-dependent and mitochondria-independent pathways of apoptosis," Archives of Toxicology, vol. 87, no. 7, pp. 1157-1180, 2013.

[31] A. Hanikoglu, H. Ozben, F. Hanikoglu, and T. Ozben, "Hybrid compounds \& oxidative stress induced apoptosis in cancer therapy," Current Medicinal Chemistry, vol. 27, no. 13, pp. 2118-2132, 2020.

[32] Y. Y. Yu, J. Lu, K. Oliphant, N. Gupta, K. Claud, and L. Lu, "Maternal administration of probiotics promotes gut development in mouse offsprings," PLoS One, vol. 15, no. 8, article e0237182, 2020.

[33] C. R. Reczek and N. S. Chandel, "ROS-dependent signal transduction," Current Opinion in Cell Biology, vol. 33, pp. 8-13, 2015.

[34] G. S. Shadel and T. L. Horvath, "Mitochondrial ROS signaling in organismal homeostasis," Cell, vol. 163, no. 3, pp. 560-569, 2015.

[35] S. Banerjee, S. Ghosh, A. Mandal, N. Ghosh, and P. C. Sil, "ROS-associated immune response and metabolism: a mechanistic approach with implication of various diseases," Archives of Toxicology, vol. 94, no. 7, pp. 2293-2317, 2020.

[36] G. Pizzino, N. Irrera, M. Cucinotta et al., "Oxidative stress: harms and benefits for human health," Oxidative Medicine and Cellular Longevity, vol. 2017, Article ID 8416763, 13 pages, 2017.

[37] L. Flohé, "The fairytale of the GSSG/GSH redox potential," Biochimica et Biophysica Acta, vol. 1830, no. 5, pp. 31393142, 2013.

[38] K. M. Holmström and T. Finkel, "Cellular mechanisms and physiological consequences of redox-dependent signalling," Nature Reviews Molecular Cell Biology, vol. 15, no. 6, pp. 411-421, 2014.

[39] S. Orrenius, V. Gogvadze, and B. Zhivotovsky, "Mitochondrial oxidative stress: implications for cell death," Annual Review of Pharmacology and Toxicology, vol. 47, no. 1, pp. 143-183, 2007.

[40] J. D. Ly, D. R. Grubb, and A. Lawen, "The mitochondrial membrane potential (deltapsi(m)) in apoptosis; an update," Apoptosis, vol. 8, no. 2, pp. 115-128, 2003.

[41] W. Li, J. Ji, X. Chen, M. Jiang, X. Rui, and M. Dong, "Structural elucidation and antioxidant activities of exopolysaccharides from Lactobacillus helveticus MB2-1," Carbohydrate Polymers, vol. 102, pp. 351-359, 2014.

[42] C. Tomaro-Duchesneau, S. Saha, M. Malhotra et al., "Effect of orally administered L. fermentum NCIMB 5221 on markers of metabolic syndrome: an in vivo analysis using ZDF rats," Applied Microbiology and Biotechnology, vol. 98, no. 1, pp. 115-126, 2014.

[43] A. Finamore, R. Ambra, F. Nobili, I. Garaguso, A. Raguzzini, and M. Serafini, "Redox role of Lactobacillus casei Shirota against the cellular damage induced by $2,2^{\prime}$-Azobis (2- 
amidinopropane) dihydrochloride-induced oxidative and inflammatory stress in enterocytes-like epithelial cells," Frontiers in Immunology, vol. 9, article 1131, 2018.

[44] G. Bartosz, "Non-enzymatic antioxidant capacity assays: limitations of use in biomedicine," Free Radical Research, vol. 44, no. 7, pp. 711-720, 2010.

[45] I. Peluso and A. Raguzzini, "Salivary and urinary total antioxidant capacity as biomarkers of oxidative stress in humans," Pathology Research International, vol. 2016, Article ID 5480267, 14 pages, 2016.

[46] S. W. Ryter and A. M. K. Choi, "Heme oxygenase-1: molecular mechanisms of gene expression in oxygen-related stress," Antioxidant \& Redox Signaling, vol. 4, no. 4, pp. 625-632, 2002.

[47] H. Yang and T. H. Lee, "Antioxidant enzymes as redox-based biomarkers: a brief review," BMB Reports, vol. 48, no. 4, pp. 200-208, 2015.

[48] X. G. Lei, J. H. Zhu, W. H. Cheng et al., "Paradoxical roles of antioxidant enzymes: basic mechanisms and health implications," Physiological Reviews, vol. 96, no. 1, pp. 307-364, 2016.

[49] G. Devi, R. Harikrishnan, B. A. Paray, M. K. al-Sadoon, S. H. Hoseinifar, and C. Balasundaram, "Effect of symbiotic supplemented diet on innate-adaptive immune response, cytokine gene regulation and antioxidant property in Labeo rohita against Aeromonas hydrophila," Fish \& Shellfish Immunology, vol. 89, pp. 687-700, 2019.

[50] F. Chen, H. Wang, J. Chen et al., "Lactobacillus delbrueckii ameliorates intestinal integrity and antioxidant ability in weaned piglets after a Lipopolysaccharide challenge," Oxidative Medicine and Cellular Longevity, vol. 2020, Article ID 6028606, 10 pages, 2020.

[51] S. Noureen, A. Riaz, M. Arshad, and N. Arshad, "In vitro selection and in vivo confirmation of the antioxidant ability of Lactobacillus brevis MG000874," Journal of Applied Microbiology, vol. 126, no. 4, pp. 1221-1232, 2019.

[52] Y. Zhang, R. du, L. Wang, and H. Zhang, "The antioxidative effects of probiotic Lactobacillus casei Zhang on the hyperlipidemic rats," European Food Research and Technology, vol. 231, no. 1, pp. 151-158, 2010.

[53] V. O. Tkachev, E. B. Menshchikova, and N. K. Zenkov, "Mechanism of the Nrf2/Keap1/ARE signaling system," Biochemistry, vol. 76, no. 4, pp. 407-422, 2011.

[54] Q. Ma, "Role of Nrf2 in oxidative stress and toxicity," Annual Review of Pharmacology and Toxicology, vol. 53, no. 1, pp. 401-426, 2013.

[55] T. Suzuki and M. Yamamoto, "Molecular basis of the Keap1Nrf2 system," Free Radical Biology and Medicine, vol. 88, Part B, pp. 93-100, 2015.

[56] D. Gao, Z. Gao, and G. Zhu, "Antioxidant effects of Lactobacillus plantarum via activation of transcription factor Nrf2," Food \& Function, vol. 4, no. 6, pp. 982-989, 2013.

[57] R. Chauhan, A. Sudhakaran Vasanthakumari, H. Panwar et al., "Amelioration of colitis in mouse model by exploring antioxidative potentials of an indigenous probiotic strain of Lactobacillus fermentum Lf1," Biomed Research International, vol. 2014, Article ID 206732, 12 pages, 2014.

[58] M. Kobayashi and M. Yamamoto, "Molecular mechanisms activating the Nrf2-Keap1 pathway of antioxidant gene regulation," Antioxidants \& Redox Signaling, vol. 7, no. 3-4, pp. 385394, 2005.
[59] E. Kobatake, H. Nakagawa, T. Seki, and T. Miyazaki, "Protective effects and functional mechanisms of Lactobacillus gasseri SBT2055 against oxidative stress," PLoS One, vol. 12, no. 5, article e0177106, 2017.

[60] Y. Liu, Q. Liu, J. Hesketh et al., "Protective effects of seleniumglutathione-enriched probiotics on $\mathrm{CCl}_{4}$-induced liver fibrosis," The Journal of Nutritional Biochemistry, vol. 58, pp. 138-149, 2018.

[61] A. Seth, F. Yan, D. B. Polk, and R. K. Rao, "Probiotics ameliorate the hydrogen peroxide-induced epithelial barrier disruption by a PKC- and MAP kinase-dependent mechanism," American Journal of Physiology-Gastrointestinal and Liver Physiology, vol. 294, no. 4, pp. G1060-G1069, 2008. 